\title{
Generalized Nonlinear Volterra-Fredholm Type Integral Inequality with Two Variables
}

\author{
Yusong Lu, ${ }^{1}$ Wu-Sheng Wang, ${ }^{1}$ Xiaoliang Zhou, ${ }^{2}$ and Yong Huang ${ }^{3}$ \\ ${ }^{1}$ School of Mathematics and Statistics, Hechi University, Yizhou, Guangxi 546300, China \\ ${ }^{2}$ Department of Mathematics, Zhanjiang Normal University, Zhanjiang, Guangdong 524088, China \\ ${ }^{3}$ Department of Mathematics and Computer Information Engineering, Baise University, Baise, Guangxi 533000, China
}

Correspondence should be addressed to Wu-Sheng Wang; wang4896@126.com

Received 22 November 2013; Accepted 9 January 2014; Published 24 April 2014

Academic Editor: Maoan Han

Copyright (C) 2014 Yusong Lu et al. This is an open access article distributed under the Creative Commons Attribution License, which permits unrestricted use, distribution, and reproduction in any medium, provided the original work is properly cited.

\begin{abstract}
We establish a class of new nonlinear retarded Volterra-Fredholm type integral inequalities, with two variables, where known function $w$ in integral functions in Q.-H. Ma and J. Pečarić, 2008 is changed into the functions $w_{1}, w_{2}$. By adopting novel analysis techniques, such as change of variable, amplification method, differential and integration, inverse function, and the dialectical relationship between constants and variables, the upper bounds of the embedded unknown functions are estimated. The derived results can be applied in the study of solutions of ordinary differential equations and integral equations.
\end{abstract}

\section{Introduction}

Gronwall-Bellman inequality $[1,2]$ is an important tool in the study of existence, uniqueness, boundedness, oscillation, stability, and other qualitative properties of solutions of differential equations and integral equation. There can be found a lot of its generalizations in various cases from the literature (e.g., [3-6]).

Gronwall-Bellman inequality $[1,2]$ can be stated as follows. If $u$ and $f$ are nonnegative continuous functions on an interval $[a, b]$ satisfying

$$
u(t) \leq c+\int_{a}^{t} f(s) u(s) d s, \quad t \in[a, b]
$$

for some constant $c \geq 0$, then

$$
u(t) \leq c \exp \left(\int_{a}^{t} f(s) d s\right), \quad t \in[a, b]
$$

During the past few years, some investigators have established a lot of useful and interesting integral inequalities in order to achieve various goals; see [7-25] and the references cited therein.
In 2004, Pachpatte [8] has established the linear VolterraFredholm type integral inequality with retardation

$$
\begin{aligned}
u(t) \leq & k+\int_{\alpha\left(t_{0}\right)}^{\alpha(t)} a(t, s) \\
& \times\left[f(s) u(s)+\int_{\alpha\left(t_{0}\right)}^{s} c(s, \tau) u(\tau) d \tau\right] d s \\
& +\int_{\alpha\left(t_{0}\right)}^{\alpha(T)} b(t, s) u(s) d s, \quad \forall t \in I .
\end{aligned}
$$

In 2005, Agarwal et al. [9] investigated the inequality

$$
u(t) \leq a(t)+\sum_{i=1}^{n} \int_{b_{i}\left(t_{0}\right)}^{b_{i}(t)} g_{i}(t, s) w_{i}(u(s)) d s, \quad t_{0} \leq t<t_{1} .
$$

In 2006, Cheung [10] studied the inequality

$$
\begin{aligned}
u^{p}(x, y) \leq & a+\frac{p}{p-q} \\
& \times \int_{b_{1}\left(x_{0}\right)}^{b_{1}(x)} \int_{c_{1}\left(y_{0}\right)}^{c_{1}(y)} g_{1}(s, t) u^{q}(s, t) d t d s
\end{aligned}
$$




$$
\begin{array}{r}
+\frac{p}{p-q} \int_{b_{2}\left(x_{0}\right)}^{b_{2}(x)} \int_{\mathcal{C}_{2}\left(y_{0}\right)}^{c_{2}(y)} g_{2}(s, t) u^{q}(s, t) \\
\times \psi(u(s, t)) d t d s .
\end{array}
$$

In 2008, Ma and Pečarić [13] have discussed the following useful nonlinear Volterra-Fredholm type integral inequality with retardation

$$
\begin{aligned}
& u(x, y) \\
& \leq k+\int_{\alpha\left(x_{0}\right)}^{\alpha(x)} \int_{\beta\left(y_{0}\right)}^{\beta(y)} h_{1}(s, t) \\
& \times[f(s, t) w(u(s, t)) \\
& +\int_{\alpha\left(x_{0}\right)}^{s} \int_{\beta\left(y_{0}\right)}^{t} h_{2}(\tau, \xi) w \\
& +\int_{\alpha\left(x_{0}\right)}^{\alpha(M)} \int_{\beta\left(y_{0}\right)}^{\beta(N)} h_{1}(s, t) \\
& \times[f(s, t) w(u(s, t))
\end{aligned}
$$$$
u(x, y) \leq k+\int_{\alpha\left(x_{0}\right)}^{\alpha(x)} \int_{\beta\left(y_{0}\right)}^{\beta(y)} h_{1}\left(s_{1}, t_{1}\right) w\left(u\left(s_{1}, t_{1}\right)\right)
$$$$
\times\left[f_{1}\left(s_{1}, t_{1}\right) w_{1}\left(u\left(s_{1}, t_{1}\right)\right)\right.
$$$$
+\int_{\alpha\left(x_{0}\right)}^{s_{1}} \int_{\beta\left(y_{0}\right)}^{t_{1}} h_{2}\left(s_{2}, t_{2}\right)
$$$$
\times\left[f_{2}\left(s_{2}, t_{2}\right) w_{2}\left(u\left(s_{2}, t_{2}\right)\right)\right.
$$$$
\left.\left.+\int_{\alpha\left(x_{0}\right)}^{s_{2}} \int_{\beta\left(y_{0}\right)}^{t_{2}} h_{3}\left(s_{3}, t_{3}\right) w_{3}\left(u\left(s_{3}, t_{3}\right)\right) d t_{3} d s_{3}\right] d t_{2} d s_{2}\right] d t_{1} d s_{1}
$$$$
+\int_{\alpha\left(x_{0}\right)}^{\alpha(M)} \int_{\beta\left(y_{0}\right)}^{\beta(N)} h_{1}\left(s_{1}, t_{1}\right) w\left(u\left(s_{1}, t_{1}\right)\right)
$$$$
\times\left[f_{1}\left(s_{1}, t_{1}\right) w_{1}\left(u\left(s_{1}, t_{1}\right)\right)\right.
$$$$
+\int_{\alpha\left(x_{0}\right)}^{s_{1}} \int_{\beta\left(y_{0}\right)}^{t_{1}} h_{2}\left(s_{2}, t_{2}\right)
$$$$
\times\left[f_{2}\left(s_{2}, t_{2}\right) w_{2}\left(u\left(s_{2}, t_{2}\right)\right)\right.
$$$$
\left.\left.+\int_{\alpha\left(x_{0}\right)}^{s_{2}} \int_{\beta\left(y_{0}\right)}^{t_{2}} h_{3}\left(s_{3}, t_{3}\right) w_{3}\left(u\left(s_{3}, t_{3}\right)\right) d t_{3} d s_{3}\right] d t_{2} d s_{2}\right] d t_{1} d s_{1},
$$

In 2011, Abdeldaim and Yakout [20] studied a new integral inequality of Gronwall-Bellman-Pachpatte type

$$
\begin{aligned}
& u(t) \leq u_{0} \\
&+ \int_{\alpha\left(t_{0}\right)}^{t} f(s) u(s) \\
& \times {\left[u(s)+\int_{\alpha\left(t_{0}\right)}^{s} h(\tau)\right.} \\
& \times {\left[u(\tau)+\int_{\alpha\left(t_{0}\right)}^{\tau} g(\xi)\right.} \\
&\times u(\xi) d \xi] d \tau] d s .
\end{aligned}
$$

In this paper, on the basis of $[13,20]$, we discuss a new retarded nonlinear Volterra-Fredholm type integral inequality 
where $k$ is a constant, where the known function $w$ in integral functions in [13] is replaced to the functions $w_{1}, w_{2}$. The upper bound estimation of the unknown function is given by integral inequality technique. Furthermore, we apply our result to retarded nonlinear Volterra-Fredholm type equations for estimation.

\section{Main Result}

Throughout this paper, $I_{1}=\left[x_{0}, M\right]$ and $I_{2}=\left[y_{0}, N\right]$ are the given subsets of real numbers $\mathbf{R}, \mathbf{R}_{+}=[0,+\infty), \Delta=I_{1} \times$ $I_{2}, C^{1}(M, S)$ denotes the class of continuously differentiable functions defined on set $M$ with range in the set $S, C(M, S)$ denotes the class of continuous functions defined on set $M$ with range in the set $S$, and $\alpha^{\prime}(t)$ denotes the derived function of a function $\alpha^{\prime}(t)$.

Theorem 1. Suppose that $k>0$ is a constant; functions $u(x, y), h_{3}(x, y), f_{i}(x, y), h_{i}(x, y) \in C\left(\Delta, \mathbf{R}_{+}\right),(i=1,2)$, both $\alpha \in C^{1}\left(I_{1}, I_{1}\right)$ and $\beta \in C^{1}\left(I_{2}, I_{2}\right)$ are nondecreasing with $\alpha(x) \leq x$ on $I_{1}, \beta(y) \leq y$ on $I_{2}, w, w_{1}, w_{2} / w_{1}, w_{3} / w_{1}, w_{3} / w_{2} \in$ $C\left(\mathbf{R}_{+}, \mathbf{R}_{+}\right)$are nondecreasing functions with $w_{i}(u)>0(i=$ $1,2,3)$ for $u>0$,

$$
\begin{aligned}
& W_{1}(z)=\int_{c}^{z} \frac{d s}{w(s) w_{1}(s)}, \\
& c>0, \quad z \in(0,+\infty), \quad W_{1}(+\infty)=+\infty, \\
& W_{2}(z)=\int_{c}^{z} \frac{w_{1}\left(W_{1}^{-1}(s)\right) d s}{w_{2}\left(W_{1}^{-1}(s)\right)}, \\
& c>0, \quad z \in(0,+\infty), \quad W_{2}(+\infty)=+\infty,
\end{aligned}
$$

$$
\begin{gathered}
u(x, y) \leq W_{1}^{-1}\left\{W _ { 2 } ^ { - 1 } \left\{W _ { 3 } ^ { - 1 } \left\{W _ { 3 } \left\{W_{2}\left\{W_{1}(c)+\int_{\alpha\left(x_{0}\right)}^{\alpha(x)} \int_{\beta\left(y_{0}\right)}^{\beta(y)} h_{1}\left(s_{1}, t_{1}\right) f_{1}\left(s_{1}, t_{1}\right) d t_{1} d s_{1}\right\}\right.\right.\right.\right. \\
\left.\quad+\int_{\alpha\left(x_{0}\right)}^{\alpha(x)} \int_{\beta\left(y_{0}\right)}^{\beta(y)} h_{1}\left(s_{1}, t_{1}\right)\left[\int_{\alpha\left(x_{0}\right)}^{s_{1}} \int_{\beta\left(y_{0}\right)}^{t_{1}} h_{2}\left(s_{2}, t_{2}\right) f_{2}\left(s_{2}, t_{2}\right) d t_{2} d s_{2}\right] d t_{1} d s_{1}\right\} \\
+\int_{\alpha\left(x_{0}\right)}^{\alpha(x)} \int_{\beta\left(y_{0}\right)}^{\beta(y)} h_{1}\left(s_{1}, t_{1}\right) \\
\times\left[\int_{\alpha\left(x_{0}\right)}^{s_{1}} \int_{\beta\left(y_{0}\right)}^{t_{1}} h_{2}\left(s_{2}, t_{2}\right)\right. \\
\left.\left.\left.\left.\times\left[\int_{\alpha\left(x_{0}\right)}^{s_{2}} \int_{\beta\left(y_{0}\right)}^{t_{2}} h_{3}\left(s_{3}, t_{3}\right) d t_{3} d s_{3}\right] d t_{2} d s_{2}\right] d t_{1} d s_{1}\right\}\right\}\right\}, \\
\forall(x, y) \in \Delta,
\end{gathered}
$$

where $W_{i}^{-1}(i=1,2,3)$ are inverse functions of $W_{i}$, respectively. 
Proof. Let $z_{1}(x, y)$ denote the function on the right-hand side of (8), which is positive and nondecreasing in each of the variables $(x, y) \in \Delta$. From (8), we have

$$
u(x, y) \leq z_{1}(x, y), \quad \forall(x, y) \in \Delta,
$$

$$
\begin{aligned}
& z_{1}\left(x_{0}, y\right)=k+\int_{\alpha\left(x_{0}\right)}^{\alpha(M)} \int_{\beta\left(y_{0}\right)}^{\beta(N)} h_{1}\left(s_{1}, t_{1}\right) w\left(u\left(s_{1}, t_{1}\right)\right) \\
& \times\left[f_{1}\left(s_{1}, t_{1}\right) w_{1}\left(u\left(s_{1}, t_{1}\right)\right)\right. \\
& +\int_{\alpha\left(x_{0}\right)}^{s_{1}} \int_{\beta\left(y_{0}\right)}^{t_{1}} h_{2}\left(s_{2}, t_{2}\right) \\
& \times\left[f_{2}\left(s_{2}, t_{2}\right) w_{2}\left(u\left(s_{2}, t_{2}\right)\right)\right. \\
& \quad+\int_{\alpha\left(x_{0}\right)}^{s_{2}} \int_{\beta\left(y_{0}\right)}^{t_{2}} h_{3}\left(s_{3}, t_{3}\right) \\
& \left.\left.\times w_{3}\left(u\left(s_{3}, t_{3}\right)\right) d t_{3} d s_{3}\right] d t_{2} d s_{2}\right] d t_{1} d s_{1} .
\end{aligned}
$$

Differentiating $z_{1}(x, y)$ with respect to $x$, using (14), we have

$$
\begin{gathered}
\frac{\partial}{\partial x} z_{1}(x, y) \\
=\alpha^{\prime}(x) \int_{\beta\left(y_{0}\right)}^{\beta(y)} h_{1}\left(\alpha(x), t_{1}\right) w\left(u\left(\alpha(x), t_{1}\right)\right) \\
\times\left[f_{1}\left(\alpha(x), t_{1}\right) w_{1}\left(u\left(\alpha(x), t_{1}\right)\right)\right. \\
+\int_{\alpha\left(x_{0}\right)}^{\alpha(x)} \int_{\beta\left(y_{0}\right)}^{t_{1}} h_{2}\left(s_{2}, t_{2}\right) \\
\times\left[f_{2}\left(s_{2}, t_{2}\right) w_{2}\left(u\left(s_{2}, t_{2}\right)\right)\right. \\
+\int_{\alpha\left(x_{0}\right)}^{s_{2}} \int_{\beta\left(y_{0}\right)}^{t_{2}} h_{3}\left(s_{3}, t_{3}\right) \\
\left.\left.\times w_{3}\left(u\left(s_{3}, t_{3}\right)\right) d t_{3} d s_{3}\right] d t_{2} d s_{2}\right] d t_{1}
\end{gathered}
$$$$
\leq \alpha^{\prime}(x) \int_{\beta\left(y_{0}\right)}^{\beta(y)} h_{1}\left(\alpha(x), t_{1}\right) w\left(z_{1}\left(\alpha(x), t_{1}\right)\right)
$$$$
\times\left[f_{1}\left(\alpha(x), t_{1}\right) w_{1}\left(z_{1}\left(\alpha(x), t_{1}\right)\right)\right.
$$$$
+\int_{\alpha\left(x_{0}\right)}^{\alpha(x)} \int_{\beta\left(y_{0}\right)}^{t_{1}} h_{2}\left(s_{2}, t_{2}\right)
$$

$$
\times\left[f_{2}\left(s_{2}, t_{2}\right) w_{2}\left(z_{1}\left(s_{2}, t_{2}\right)\right)\right.
$$$$
+\int_{\alpha\left(x_{0}\right)}^{s_{2}} \int_{\beta\left(y_{0}\right)}^{t_{2}} h_{3}\left(s_{3}, t_{3}\right) d t_{1}
$$$$
\left.\left.\times w_{3}\left(z_{1}\left(s_{3}, t_{3}\right)\right) d t_{3} d s_{3}\right] d t_{2} d s_{2}\right]
$$

by the monotonicity of $w, w_{1}, w_{2}, w_{3}$, and $z_{1}$ and the property of $\alpha, \beta$. From (16), we have

$$
\begin{gathered}
\frac{(\partial / \partial x) z_{1}(x, y)}{w\left(z_{1}(x, y)\right) w_{1}\left(z_{1}(x, y)\right)} \\
\leq \alpha^{\prime}(x) \int_{\beta\left(y_{0}\right)}^{\beta(y)} h_{1}\left(\alpha(x), t_{1}\right)
\end{gathered}
$$

$$
\times\left[f_{1}\left(\alpha(x), t_{1}\right)\right.
$$$$
+\int_{\alpha\left(x_{0}\right)}^{\alpha(x)} \int_{\beta\left(y_{0}\right)}^{t_{1}} h_{2}\left(s_{2}, t_{2}\right)
$$

$$
\times\left[f_{2}\left(s_{2}, t_{2}\right) \frac{w_{2}\left(z_{1}\left(s_{2}, t_{2}\right)\right)}{w_{1}\left(z_{1}\left(s_{2}, t_{2}\right)\right)}\right.
$$




$$
\begin{aligned}
& +\int_{\alpha\left(x_{0}\right)}^{s_{2}} \int_{\beta\left(y_{0}\right)}^{t_{2}} h_{3}\left(s_{3}, t_{3}\right) \\
& \left.\left.\quad \times \frac{w_{3}\left(z_{1}\left(s_{3}, t_{3}\right)\right)}{w_{1}\left(z_{1}\left(s_{3}, t_{3}\right)\right)} d t_{3} d s_{3}\right] d t_{2} d s_{2}\right] d t_{1} .
\end{aligned}
$$

Integrating both sides of the above inequality from $x_{0}$ to $x$, we obtain

$$
\begin{aligned}
& W_{1}\left(z_{1}(x, y)\right) \\
& \leq W_{1}\left(z_{1}\left(x_{0}, y\right)\right) \\
& +\int_{\alpha\left(x_{0}\right)}^{\alpha(x)} \int_{\beta\left(y_{0}\right)}^{\beta(y)} h_{1}\left(s_{1}, t_{1}\right) \\
& \times\left[f_{1}\left(s_{1}, t_{1}\right)\right. \\
& +\int_{\alpha\left(x_{0}\right)}^{s_{1}} \int_{\beta\left(y_{0}\right)}^{t_{1}} h_{2}\left(s_{2}, t_{2}\right) \\
& \times\left[f_{2}\left(s_{2}, t_{2}\right) \frac{w_{2}\left(z_{1}\left(s_{2}, t_{2}\right)\right)}{w_{1}\left(z_{1}\left(s_{2}, t_{2}\right)\right)}\right. \\
& +\int_{\alpha\left(x_{0}\right)}^{s_{2}} \int_{\beta\left(y_{0}\right)}^{t_{2}} h_{3}\left(s_{3}, t_{3}\right) \\
& \left.\left.\times \frac{w_{3}\left(z_{1}\left(s_{3}, t_{3}\right)\right)}{w_{1}\left(z_{1}\left(s_{3}, t_{3}\right)\right)} d t_{3} d s_{3}\right] d t_{2} d s_{2}\right] d t_{1} d s_{1}
\end{aligned}
$$$$
\leq W_{1}\left(z_{1}\left(x_{0}, y\right)\right)
$$$$
+\int_{\alpha\left(x_{0}\right)}^{\alpha(X)} \int_{\beta\left(y_{0}\right)}^{\beta(Y)} h_{1}\left(s_{1}, t_{1}\right) f_{1}\left(s_{1}, t_{1}\right) d t_{1} d s_{1}
$$$$
+\int_{\alpha\left(x_{0}\right)}^{\alpha(x)} \int_{\beta\left(y_{0}\right)}^{\beta(y)} h_{1}\left(s_{1}, t_{1}\right)
$$$$
\times\left[\int_{\alpha\left(x_{0}\right)}^{s_{1}} \int_{\beta\left(y_{0}\right)}^{t_{1}} h_{2}\left(s_{2}, t_{2}\right)\right.
$$$$
\times\left[f_{2}\left(s_{2}, t_{2}\right) \frac{w_{2}\left(z_{1}\left(s_{2}, t_{2}\right)\right)}{w_{1}\left(z_{1}\left(s_{2}, t\right)\right)}\right.
$$$$
+\int_{\alpha\left(x_{0}\right)}^{s_{2}} \int_{\beta\left(y_{0}\right)}^{t_{2}} h_{3}\left(s_{3}, t_{3}\right)
$$$$
\left.\left.\times \frac{w_{3}\left(z_{1}\left(s_{3}, t_{3}\right)\right)}{w_{1}\left(z_{1}\left(s_{3}, t_{3}\right)\right)} d t_{3} d s_{3}\right] d t_{2} d s_{2}\right] d t_{1} d s_{1},
$$

for all $(x, y) \in\left[x_{0}, X\right] \times\left[y_{0}, Y\right], X \in I_{1}, Y \in I_{2}$, and $X, Y$ are chosen arbitrarily, where $W_{1}$ is defined by (9).
Let $z_{2}(x, y)$ denote the function on the right-hand side of (18), which is positive and nondecreasing in each of the variables $(x, y) \in\left[x_{0}, X\right] \times\left[y_{0}, Y\right]$. From (18), we have

$$
z_{1}(x, y) \leq W_{1}^{-1}\left(z_{2}(x, y)\right), \quad \forall(x, y) \in\left[x_{0}, X\right] \times\left[y_{0}, Y\right]
$$

$$
\begin{aligned}
z_{2}\left(x_{0}, y\right)= & W_{1}\left(z_{1}\left(x_{0}, y\right)\right) \\
& +\int_{\alpha\left(x_{0}\right)}^{\alpha(X)} \int_{\beta\left(y_{0}\right)}^{\beta(Y)} h_{1}\left(s_{1}, t_{1}\right) f_{1}\left(s_{1}, t_{1}\right) d t_{1} d s_{1} .
\end{aligned}
$$

Differentiating $z_{2}(x, y)$ with respect to $x$, by the monotonicity of $z_{2}, W_{1}^{-1}, w_{2} / w_{1}$, and $w_{3} / w_{1}$, the property of $\alpha, \beta$, and (19), we have

$$
\begin{aligned}
& \frac{\partial}{\partial x} z_{2}(x, y) \\
& =\alpha^{\prime}(x) \int_{\beta\left(y_{0}\right)}^{\beta(y)} h_{1}\left(\alpha(x), t_{1}\right) \\
& \times\left[\int_{\alpha\left(x_{0}\right)}^{\alpha(x)} \int_{\beta\left(y_{0}\right)}^{t_{1}} h_{2}\left(s_{2}, t_{2}\right)\right. \\
& \times\left[f_{2}\left(s_{2}, t_{2}\right) \frac{w_{2}\left(z_{1}\left(s_{2}, t_{2}\right)\right)}{w_{1}\left(z_{1}\left(s_{2}, t_{2}\right)\right)}\right. \\
& +\int_{\alpha\left(x_{0}\right)}^{s_{2}} \int_{\beta\left(y_{0}\right)}^{t_{2}} h_{3}\left(s_{3}, t_{3}\right) \\
& \left.\left.\times \frac{w_{3}\left(z_{1}\left(s_{3}, t_{3}\right)\right)}{w_{1}\left(z_{1}\left(s_{3}, t_{3}\right)\right)} d t_{3} d s_{3}\right] d t_{2} d s_{2}\right] d t_{1} \\
& \leq \alpha^{\prime}(x) \int_{\beta\left(y_{0}\right)}^{\beta(y)} h_{1}\left(\alpha(x), t_{1}\right) \\
& \times\left[\int_{\alpha\left(x_{0}\right)}^{\alpha(x)} \int_{\beta\left(y_{0}\right)}^{t_{1}} h_{2}\left(s_{2}, t_{2}\right)\right. \\
& \times\left[f_{2}\left(s_{2}, t_{2}\right) \frac{w_{2}\left(W_{1}^{-1}\left(z_{2}\left(s_{2}, t_{2}\right)\right)\right)}{w_{1}\left(W_{1}^{-1}\left(z_{2}\left(s_{2}, t_{1}\right)\right)\right)}\right. \\
& +\int_{\alpha\left(x_{0}\right)}^{s_{2}} \int_{\beta\left(y_{0}\right)}^{t_{2}} h_{3}\left(s_{3}, t_{3}\right) \\
& \left.\left.\times \frac{w_{3}\left(W_{1}^{-1}\left(z_{2}\left(s_{3}, t_{3}\right)\right)\right)}{w_{1}\left(W_{1}^{-1}\left(z_{2}\left(s_{3}, t_{3}\right)\right)\right)} d t_{3} d s_{3}\right] d t_{2} d s_{2}\right] d t_{1}
\end{aligned}
$$


for all $(x, y) \in\left[x_{0}, X\right] \times\left[y_{0}, Y\right]$. From (21), we have

$$
\begin{gathered}
\frac{w_{1}\left(W_{1}^{-1}\left(z_{2}(x, y)\right)\right)(\partial / \partial x) z_{2}(x, y)}{w_{2}\left(W_{1}^{-1}\left(z_{2}(x, y)\right)\right)} \\
\leq \alpha^{\prime}(x) \int_{\beta\left(y_{0}\right)}^{\beta(y)} h_{1}\left(\alpha(x), t_{1}\right) \\
\times\left[\int_{\alpha\left(x_{0}\right)}^{\alpha(x)} \int_{\beta\left(y_{0}\right)}^{t_{1}} h_{2}\left(s_{2}, t_{2}\right)\right.
\end{gathered}
$$$$
\times\left[f_{2}\left(s_{2}, t_{2}\right)\right.
$$$$
+\int_{\alpha\left(x_{0}\right)}^{s_{2}} \int_{\beta\left(y_{0}\right)}^{t_{2}} h_{3}\left(s_{3}, t_{3}\right)
$$$$
\left.\left.\times \frac{w_{3}\left(W_{1}^{-1}\left(z_{2}\left(s_{3}, t_{3}\right)\right)\right)}{w_{2}\left(W_{1}^{-1}\left(z_{2}\left(s_{3}, t_{3}\right)\right)\right)} d t_{3} d s_{3}\right] d t_{2} d s_{2}\right] d t_{1},
$$

for all $(x, y) \in\left[x_{0}, X\right] \times\left[y_{0}, Y\right]$. From (22), we have

$$
\begin{aligned}
& W_{2}\left(z_{2}(x, y)\right) \leq W_{2}\left(z_{2}\left(x_{0}, y\right)\right) \\
& +\int_{\alpha\left(x_{0}\right)}^{\alpha(x)} \int_{\beta\left(y_{0}\right)}^{\beta(y)} h_{1}\left(s_{1}, t_{1}\right) \\
& \times\left[\int_{\alpha\left(x_{0}\right)}^{s_{1}} \int_{\beta\left(y_{0}\right)}^{t_{1}} h_{2}\left(s_{2}, t_{2}\right)\right. \\
& \times\left[f_{2}\left(s_{2}, t_{2}\right)\right. \\
& +\int_{\alpha\left(x_{0}\right)}^{s_{2}} \int_{\beta\left(y_{0}\right)}^{t_{2}} h_{3}\left(s_{3}, t_{3}\right) \\
& \left.\left.\times \frac{w_{3}\left(W_{1}^{-1}\left(z_{2}\left(s_{3}, t_{3}\right)\right)\right)}{w_{2}\left(W_{1}^{-1}\left(z_{2}\left(s_{3}, t_{3}\right)\right)\right)} d t_{3} d s_{3}\right] d t_{2} d s_{2}\right] d t_{1} d s_{1}
\end{aligned}
$$

$$
\begin{aligned}
& \leq W_{2}\left(z_{2}\left(x_{0}, y\right)\right) \\
& +\int_{\alpha\left(x_{0}\right)}^{\alpha(X)} \int_{\beta\left(y_{0}\right)}^{\beta(Y)} h_{1}\left(s_{1}, t_{1}\right)\left[\int_{\alpha\left(x_{0}\right)}^{s_{1}} \int_{\beta\left(y_{0}\right)}^{t_{1}} h_{2}\left(s_{2}, t_{2}\right) f_{2}\left(s_{2}, t_{2}\right) d t_{2} d s_{2}\right] d t_{1} d s_{1} \\
& +\int_{\alpha\left(x_{0}\right)}^{\alpha(x)} \int_{\beta\left(y_{0}\right)}^{\beta(y)} h_{1}\left(s_{1}, t_{1}\right) \\
& \times\left[\int_{\alpha\left(x_{0}\right)}^{s_{1}} \int_{\beta\left(y_{0}\right)}^{t_{1}} h_{2}\left(s_{2}, t_{2}\right)\right. \\
& \left.\times\left[\int_{\alpha\left(x_{0}\right)}^{s_{2}} \int_{\beta\left(y_{0}\right)}^{t_{2}} h_{3}\left(s_{3}, t_{3}\right) \frac{w_{3}\left(W_{1}^{-1}\left(z_{2}\left(s_{3}, t_{3}\right)\right)\right)}{w_{2}\left(W_{1}^{-1}\left(z_{2}\left(s_{3}, t_{3}\right)\right)\right)} d t_{3} d s_{3}\right] d t_{2} d s_{2}\right] d t_{1} d s_{1},
\end{aligned}
$$


Journal of Applied Mathematics

7

for all $(x, y) \in\left[x_{0}, X\right] \times\left[y_{0}, Y\right]$, where $W_{2}$ is defined by (10). Let $z_{3}(x, y)$ denote the function on the right-hand side of (23), which is positive and nondecreasing in each of the variables $(x, y) \in\left[x_{0}, X\right] \times\left[y_{0}, Y\right]$. Then (23) is equivalent to

$$
z_{2}(x, y) \leq W_{2}^{-1}\left(z_{3}(x, y)\right), \quad \forall(x, y) \in\left[x_{0}, X\right] \times\left[y_{0}, Y\right]
$$

$$
\begin{aligned}
& z_{3}\left(x_{0}, y\right) \\
& \quad=W_{2}\left(z_{2}\left(x_{0}, y\right)\right)
\end{aligned}
$$

$$
\begin{aligned}
+\int_{\alpha\left(x_{0}\right)}^{\alpha(X)} \int_{\beta\left(y_{0}\right)}^{\beta(Y)} h_{1}\left(s_{1}, t_{1}\right) \\
\times\left[\int_{\alpha\left(x_{0}\right)}^{s_{1}} \int_{\beta\left(y_{0}\right)}^{t_{1}} h_{2}\left(s_{2}, t_{2}\right)\right. \\
\left.\quad \times f_{2}\left(s_{2}, t_{2}\right) d t_{2} d s_{2}\right] d t_{1} d s_{1} .
\end{aligned}
$$

Differentiating $z_{3}(x, y)$ with respect to $x$, using (24), we have

$$
\begin{aligned}
\frac{\partial}{\partial x} z_{3}(x, y)=\alpha^{\prime}(x) \int_{\beta\left(y_{0}\right)}^{\beta(y)} h_{1}\left(\alpha(x), t_{1}\right) & \\
\times & \times \int_{\alpha\left(x_{0}\right)}^{\alpha(x)} \int_{\beta\left(y_{0}\right)}^{t_{1}} h_{2}\left(s_{2}, t_{2}\right) \\
& \left.\times\left[\int_{\alpha\left(x_{0}\right)}^{s_{2}} \int_{\beta\left(y_{0}\right)}^{t_{2}} h_{3}\left(s_{3}, t_{3}\right) \frac{w_{3}\left(W_{1}^{-1}\left(z_{2}\left(s_{3}, t_{3}\right)\right)\right)}{w_{2}\left(W_{1}^{-1}\left(z_{2}\left(s_{3}, t_{3}\right)\right)\right)} d t_{3} d s_{3}\right] d t_{2} d s_{2}\right] d t_{1} \\
\leq \alpha^{\prime}(x) \int_{\beta\left(y_{0}\right)}^{\beta(y)} h_{1}\left(\alpha(x), t_{1}\right) & \times\left[\int_{\alpha\left(x_{0}\right)}^{\alpha(x)} \int_{\beta\left(y_{0}\right)}^{t_{1}} h_{2}\left(s_{2}, t_{2}\right)\right. \\
\times & {\left.\left[\int_{\alpha\left(x_{0}\right)}^{s_{2}} \int_{\beta\left(y_{0}\right)}^{t_{2}} h_{3}\left(s_{3}, t_{3}\right) \frac{w_{3}\left(W_{1}^{-1}\left(W_{2}^{-1}\left(z_{3}\left(s_{3}, t_{3}\right)\right)\right)\right)}{w_{2}\left(W_{1}^{-1}\left(W_{2}^{-1}\left(z_{3}\left(s_{3}, t_{3}\right)\right)\right)\right)} d t_{3} d s_{3}\right] d t_{2} d s_{2}\right] d t_{1}, }
\end{aligned}
$$

for all $(x, y) \in\left[x_{0}, X\right] \times\left[y_{0}, Y\right]$. From (26), using the monotonicity of $z_{3}, W_{1}^{-1}, W_{2}^{-1}$, and $w_{3} / w_{2}$ and the property of $\alpha, \beta$, we have

$$
\begin{aligned}
& \frac{w_{2}\left(W_{1}^{-1}\left(W_{2}^{-1}\left(z_{3}(x, y)\right)\right)\right)(\partial / \partial x) z_{3}(x, y)}{w_{3}\left(W_{1}^{-1}\left(W_{2}^{-1}\left(z_{3}(x, y)\right)\right)\right)} \leq \alpha^{\prime}(x) \int_{\beta\left(y_{0}\right)}^{\beta(y)} h_{1}\left(\alpha(x), t_{1}\right) \times\left[\int_{\alpha\left(x_{0}\right)}^{\alpha(x)} \int_{\beta\left(y_{0}\right)}^{t_{1}} h_{2}\left(s_{2}, t_{2}\right)\right. \\
&\left.\times\left[\int_{\alpha\left(x_{0}\right)}^{s_{2}} \int_{\beta\left(y_{0}\right)}^{t_{2}} h_{3}\left(s_{3}, t_{3}\right) d t_{3} d s_{3}\right] d t_{2} d s_{2}\right] d t_{1},
\end{aligned}
$$


for all $(x, y) \in\left[x_{0}, X\right] \times\left[y_{0}, Y\right]$. Integrating both sides of the above inequality from $x_{0}$ to $x$, we obtain

$$
\begin{aligned}
& W_{3}\left(z_{3}(x, y)\right) \leq W_{3}\left(z_{3}\left(x_{0}, y\right)\right) \\
&+\int_{\alpha\left(x_{0}\right)}^{\alpha(x)} \int_{\beta\left(y_{0}\right)}^{\beta(y)} h_{1}\left(s_{1}, t_{1}\right)\left[\int_{\alpha\left(x_{0}\right)}^{s_{1}} \int_{\beta\left(y_{0}\right)}^{t_{1}} h_{2}\left(s_{2}, t_{2}\right)\left[\int_{\alpha\left(x_{0}\right)}^{s_{2}} \int_{\beta\left(y_{0}\right)}^{t_{2}} h_{3}\left(s_{3}, t_{3}\right) d t_{3} d s_{3}\right] d t_{2} d s_{2}\right] d t_{1} d s_{1}, \\
& \forall(x, y) \in\left[x_{0}, X\right] \times\left[y_{0}, Y\right]
\end{aligned}
$$

where $W_{3}$ is defined by (11).

From (19), (24), and (28), we have

$$
\begin{aligned}
& z_{1}(x, y) \leq W_{1}^{-1}\left(z_{2}(x, y)\right) \\
& \leq W_{1}^{-1}\left(W_{2}^{-1}\left(z_{3}(x, y)\right)\right) \\
& \leq W_{1}^{-1}\left\{W _ { 2 } ^ { - 1 } \left\{W _ { 3 } ^ { - 1 } \left\{W_{3}\left(z_{3}\left(x_{0}, y\right)\right)\right.\right.\right. \\
&\left.\left.\left.+\int_{\alpha\left(x_{0}\right)}^{\alpha(x)} \int_{\beta\left(y_{0}\right)}^{\beta(y)} h_{1}\left(s_{1}, t_{1}\right) \quad \times\left[\int_{\alpha\left(x_{0}\right)}^{s_{1}} \int_{\beta\left(y_{0}\right)}^{t_{1}} h_{2}\left(s_{2}, t_{2}\right)\left[\int_{\alpha\left(x_{0}\right)}^{s_{2}} \int_{\beta\left(y_{0}\right)}^{t_{2}} h_{3}\left(s_{3}, t_{3}\right) d t_{3} d s_{3}\right] d t_{2} d s_{2}\right] d t_{1} d s_{1}\right\}\right\}\right\}, \\
& \forall(x, y) \in\left[x_{0}, X\right] \times\left[y_{0}, Y\right] .
\end{aligned}
$$

Substituting (20) and (25) into (29), we have

$$
\begin{aligned}
z_{1}(x, y) \leq & W_{1}^{-1}\left(z_{2}(x, y)\right) \leq W_{1}^{-1}\left(W_{2}^{-1}\left(z_{3}(x, y)\right)\right) \\
\leq & W_{1}^{-1}\left\{W _ { 2 } ^ { - 1 } \left\{W _ { 3 } ^ { - 1 } \left\{W _ { 3 } \left\{W_{2}\left\{W_{1}\left(z_{1}\left(x_{0}, y\right)\right)+\int_{\alpha\left(x_{0}\right)}^{\alpha(X)} \int_{\beta\left(y_{0}\right)}^{\beta(Y)} h_{1}\left(s_{1}, t_{1}\right) f_{1}\left(s_{1}, t_{1}\right) d t_{1} d s_{1}\right\}\right.\right.\right.\right. \\
& \left.+\int_{\alpha\left(x_{0}\right)}^{\alpha(X)} \int_{\beta\left(y_{0}\right)}^{\beta(Y)} h_{1}\left(s_{1}, t_{1}\right)\left[\int_{\alpha\left(x_{0}\right)}^{s_{1}} \int_{\beta\left(y_{0}\right)}^{t_{1}} h_{2}\left(s_{2}, t_{2}\right) f_{2}\left(s_{2}, t_{2}\right) d t_{2} d s_{2}\right] d t_{1} d s_{1}\right\} \\
& +\int_{\alpha\left(x_{0}\right)}^{\alpha(x)} \int_{\beta\left(y_{0}\right)}^{\beta(y)} h_{1}\left(s_{1}, t_{1}\right) \quad \\
\times & {\left.\left.\left.\left[\int_{\alpha\left(x_{0}\right)}^{s_{1}} \int_{\beta\left(y_{0}\right)}^{t_{1}} h_{2}\left(s_{2}, t_{2}\right)\left[\int_{\alpha\left(x_{0}\right)}^{s_{2}} \int_{\beta\left(y_{0}\right)}^{t_{2}} h_{3}\left(s_{3}, t_{3}\right) d t_{3} d s_{3}\right] d t_{2} d s_{2}\right] d t_{1} d s_{1}\right\}\right\}\right\}, }
\end{aligned}
$$


Journal of Applied Mathematics

9

Since $X, Y$ are chosen arbitrarily, we have

$$
\begin{aligned}
& z_{1}(x, y) \leq W_{1}^{-1} \\
& \times \times W_{2}^{-1}\left\{W _ { 3 } ^ { - 1 } \left\{W _ { 3 } \left\{W_{2}\left\{W_{1}\left(z_{1}\left(x_{0}, y\right)\right)+\int_{\alpha\left(x_{0}\right)}^{\alpha(x)} \int_{\beta\left(y_{0}\right)}^{\beta(y)} h_{1}\left(s_{1}, t_{1}\right) f_{1}\left(s_{1}, t_{1}\right) d t_{1} d s_{1}\right\}\right.\right.\right. \\
&\left.\quad+\int_{\alpha\left(x_{0}\right)}^{\alpha(x)} \int_{\beta\left(y_{0}\right)}^{\beta(y)} h_{1}\left(s_{1}, t_{1}\right)\left[\int_{\alpha\left(x_{0}\right)}^{s_{1}} \int_{\beta\left(y_{0}\right)}^{t_{1}} h_{2}\left(s_{2}, t_{2}\right) f_{2}\left(s_{2}, t_{2}\right) d t_{2} d s_{2}\right] d t_{1} d s_{1}\right\} \\
&+\int_{\alpha\left(x_{0}\right)}^{\alpha(x)} \int_{\beta\left(y_{0}\right)}^{\beta(y)} h_{1}\left(s_{1}, t_{1}\right) \quad \\
&\left.\left.\left.\times\left[\int_{\alpha\left(x_{0}\right)}^{s_{1}} \int_{\beta\left(y_{0}\right)}^{t_{1}} h_{2}\left(s_{2}, t_{2}\right)\left[\int_{\alpha\left(x_{0}\right)}^{s_{2}} \int_{\beta\left(y_{0}\right)}^{t_{2}} h_{3}\left(s_{3}, t_{3}\right) d t_{3} d s_{3}\right] d t_{2} d s_{2}\right] d t_{1} d s_{1}\right\}\right\}\right\}, \\
& \forall(x, y) \in \Delta .
\end{aligned}
$$

By the definition of $z_{1}$ and (15), we have

$$
\begin{aligned}
& 2 z_{1}\left(x_{0}, y\right)-k \\
& \begin{aligned}
=k+2 \int_{\alpha\left(x_{0}\right)}^{\alpha(M)} \int_{\beta\left(y_{0}\right)}^{\beta(N)} h_{1}\left(s_{1}, t_{1}\right) w\left(u\left(s_{1}, t_{1}\right)\right) \\
\times\left[f_{1}\left(s_{1}, t_{1}\right) w_{1}\left(u\left(s_{1}, t_{1}\right)\right)\right. \\
\quad+\int_{\alpha\left(x_{0}\right)}^{s_{1}} \int_{\beta\left(y_{0}\right)}^{t_{1}} h_{2}\left(s_{2}, t_{2}\right) \\
\left.\times\left[f_{2}\left(s_{2}, t_{2}\right) w_{2}\left(u\left(s_{2}, t_{2}\right)\right)+\int_{\alpha\left(x_{0}\right)}^{s_{2}} \int_{\beta\left(y_{0}\right)}^{t_{2}} h_{3}\left(s_{3}, t_{3}\right) w_{3}\left(u\left(s_{3}, t_{3}\right)\right) d t_{3} d s_{3}\right] d t_{2} d s_{2}\right] d t_{1} d s_{1}
\end{aligned} \\
& =z_{1}(M, N) .
\end{aligned}
$$

From (31) and (32), we have

$$
\begin{aligned}
2 z_{1}\left(x_{0}, y\right)-k \leq & W_{1}^{-1} \\
\times & \times\left\{W _ { 2 } ^ { - 1 } \left\{W _ { 3 } ^ { - 1 } \left\{W _ { 3 } \left\{W_{2}\left\{W_{1}\left(z_{1}\left(x_{0}, y\right)\right)+\int_{\alpha\left(x_{0}\right)}^{\alpha(M)} \int_{\beta\left(y_{0}\right)}^{\beta(N)} h_{1}\left(s_{1}, t_{1}\right) f_{1}\left(s_{1}, t_{1}\right) d t_{1} d s_{1}\right\}\right.\right.\right.\right. \\
& \left.+\int_{\alpha\left(x_{0}\right)}^{\alpha(M)} \int_{\beta\left(y_{0}\right)}^{\beta(N)} h_{1}\left(s_{1}, t_{1}\right)\left[\int_{\alpha\left(x_{0}\right)}^{s_{1}} \int_{\beta\left(y_{0}\right)}^{t_{1}} h_{2}\left(s_{2}, t_{2}\right) f_{2}\left(s_{2}, t_{2}\right) d t_{2} d s_{2}\right] d t_{1} d s_{1}\right\}
\end{aligned}
$$




$$
\begin{aligned}
+\int_{\alpha\left(x_{0}\right)}^{\alpha(M)} \int_{\beta\left(y_{0}\right)}^{\beta(N)} h_{1}\left(s_{1}, t_{1}\right) \\
\left.\left.\left.\times\left[\int_{\alpha\left(x_{0}\right)}^{s_{1}} \int_{\beta\left(y_{0}\right)}^{t_{1}} h_{2}\left(s_{2}, t_{2}\right)\left[\int_{\alpha\left(x_{0}\right)}^{s_{2}} \int_{\beta\left(y_{0}\right)}^{t_{2}} h_{3}\left(s_{3}, t_{3}\right) d t_{3} d s_{3}\right] d t_{2} d s_{2}\right] d t_{1} d s_{1}\right\}\right\}\right\}
\end{aligned}
$$

or

$$
\begin{aligned}
& W_{3}\left\{W_{2}\left\{W_{1}\left(2 z_{1}\left(x_{0}, y\right)-k\right)\right\}\right\} \\
& \leq W_{3}\left\{W_{2}\left\{W_{1}\left(z_{1}\left(x_{0}, y\right)\right)+\int_{\alpha\left(x_{0}\right)}^{\alpha(M)} \int_{\beta\left(y_{0}\right)}^{\beta(N)} h_{1}\left(s_{1}, t_{1}\right) f_{1}\left(s_{1}, t_{1}\right) d t_{1} d s_{1}\right\}\right. \\
& \left.\quad+\int_{\alpha\left(x_{0}\right)}^{\alpha(M)} \int_{\beta\left(y_{0}\right)}^{\beta(N)} h_{1}\left(s_{1}, t_{1}\right)\left[\int_{\alpha\left(x_{0}\right)}^{s_{1}} \int_{\beta\left(y_{0}\right)}^{t_{1}} h_{2}\left(s_{2}, t_{2}\right) f_{2}\left(s_{2}, t_{2}\right) d t_{2} d s_{2}\right] d t_{1} d s_{1}\right\} \\
& \quad+\int_{\alpha\left(x_{0}\right)}^{\alpha(M)} \int_{\beta\left(y_{0}\right)}^{\beta(N)} h_{1}\left(s_{1}, t_{1}\right)\left[\int_{\alpha\left(x_{0}\right)}^{s_{1}} \int_{\beta\left(y_{0}\right)}^{t_{1}} h_{2}\left(s_{2}, t_{2}\right)\left[\int_{\alpha\left(x_{0}\right)}^{s_{2}} \int_{\beta\left(y_{0}\right)}^{t_{2}} h_{3}\left(s_{3}, t_{3}\right) d t_{3} d s_{3}\right] d t_{2} d s_{2}\right] d t_{1} d s_{1} .
\end{aligned}
$$

By the definition of $H$, the assumption of Theorem 1 , and (34), we observe that

$$
H\left(z_{1}\left(x_{0}, y\right)\right) \leq 0=H(c) .
$$

Since $H$ is increasing, from (14), (31), and (35), we have the desired estimation (13).

We consider a special case of Theorem 1. If $u(x, y)$ satisfies nonlinear Volterra-Fredholm type integral inequality with retardation,

$$
\begin{aligned}
u(x, y) \leq & \\
+ & \int_{\alpha\left(x_{0}\right)}^{\alpha(x)} \int_{\beta\left(y_{0}\right)}^{\beta(y)} h_{1}\left(s_{1}, t_{1}\right) w\left(u\left(s_{1}, t_{1}\right)\right) \\
\times & {\left[f_{1}\left(s_{1}, t_{1}\right) w_{1}\left(u\left(s_{1}, t_{1}\right)\right)+\int_{\alpha\left(x_{0}\right)}^{s_{1}} \int_{\beta\left(y_{0}\right)}^{t_{1}} h_{2}\left(s_{2}, t_{2}\right) w_{2}\left(u\left(s_{2}, t_{2}\right)\right) d t_{2} d s_{2}\right] d t_{1} d s_{1} } \\
+ & \times \int_{\alpha\left(x_{0}\right)}^{\alpha(M)} \int_{\beta\left(y_{0}\right)}^{\beta(N)} h_{1}\left(s_{1}, t_{1}\right) w\left(u\left(s_{1}, t_{1}\right)\right) \\
\times & {\left[f_{1}\left(s_{1}, t_{1}\right) w_{1}\left(u\left(s_{1}, t_{1}\right)\right)+\int_{\alpha\left(x_{0}\right)}^{s_{1}} \int_{\beta\left(y_{0}\right)}^{t_{1}} h_{2}\left(s_{2}, t_{2}\right) w_{2}\left(u\left(s_{2}, t_{2}\right)\right) d t_{2} d s_{2}\right] d t_{1} d s_{1}, } \\
& \forall(x, y) \in \Delta .
\end{aligned}
$$


Corollary 2. Let functions $u(x, y), f_{1}(x, y), h_{1}(x, y)$, $h_{2}(x, y), \alpha, \beta, w, w_{1}, w_{2} / w_{1}, W_{1}$, and $W_{2}$ and constant $k$ be as in Theorem 1. Suppose that

$$
H(u)
$$

$$
\begin{aligned}
&= W_{2}\left\{W_{1}(2 u-k)\right\} \\
&- W_{2}\left\{W_{1}(u)+\int_{\alpha\left(x_{0}\right)}^{\alpha(M)} \int_{\beta\left(y_{0}\right)}^{\beta(N)} h_{1}\left(s_{1}, t_{1}\right) f_{1}\left(s_{1}, t_{1}\right) d t_{1} d s_{1}\right\} \\
&-\int_{\alpha\left(x_{0}\right)}^{\alpha(M)} \int_{\beta\left(y_{0}\right)}^{\beta(N)} h_{1}\left(s_{1}, t_{1}\right) \\
& \times\left[\int_{\alpha\left(x_{0}\right)}^{s_{1}} \int_{\beta\left(y_{0}\right)}^{t_{1}} h_{2}\left(s_{2}, t_{2}\right) d t_{2} d s_{2}\right] d t_{1} d s_{1}
\end{aligned}
$$

is increasing and $H(u)=0$ has a solution $c$ for $u>k$. If $u(x, y)$ satisfies (36), then

$u(x, y)$

$\leq W_{1}^{-1}\left\{W_{2}^{-1}\left\{W_{2}\left\{W_{1}(c)\right.\right.\right.$

$$
\begin{gathered}
+\int_{\alpha\left(x_{0}\right)}^{\alpha(x)} \int_{\beta\left(y_{0}\right)}^{\beta(y)} h_{1}\left(s_{1}, t_{1}\right) \\
\left.\times f_{1}\left(s_{1}, t_{1}\right) d t_{1} d s_{1}\right\} \\
+\int_{\alpha\left(x_{0}\right)}^{\alpha(x)} \int_{\beta\left(y_{0}\right)}^{\beta(y)} h_{1}\left(s_{1}, t_{1}\right) \\
\left.\left.\times\left[\int_{\alpha\left(x_{0}\right)}^{s_{1}} \int_{\beta\left(y_{0}\right)}^{t_{1}} h_{2}\left(s_{2}, t_{2}\right) d t_{2} d s_{2}\right] d t_{1} d s_{1}\right\}\right\} .
\end{gathered}
$$

for all $(x, y) \in \Delta$, where $W_{i}^{-1}(i=1,2)$ are inverse functions of $W_{i}$, respectively.

Remark 3. In Corollary 2, when $w_{1}(x, y) \equiv w_{2}(x, y)$ on $\Delta$, $W_{2}(u)=u-c$, and $W_{2}^{-1}(u)=u+c$, (38) is equivalent to

$$
\begin{aligned}
& u(x, y) \leq W_{1}^{-1} \\
& \times\left\{W_{1}(c)+\int_{\alpha\left(x_{0}\right)}^{\alpha(x)} \int_{\beta\left(y_{0}\right)}^{\beta(y)} h_{1}\left(s_{1}, t_{1}\right) f_{1}\left(s_{1}, t_{1}\right) d t_{1} d s_{1}\right. \\
&\left.\quad+\int_{\alpha\left(x_{0}\right)}^{\alpha(x)} \int_{\beta\left(y_{0}\right)}^{\beta(y)} h_{1}\left(s_{1}, t_{1}\right)\left[\int_{\alpha\left(x_{0}\right)}^{s_{1}} \int_{\beta\left(y_{0}\right)}^{t_{1}} h_{2}\left(s_{2}, t_{2}\right) d t_{2} d s_{2}\right] d t_{1} d s_{1}\right\} \\
&= W_{1}^{-1}\left\{W_{1}(c)+\int_{\alpha\left(x_{0}\right)}^{\alpha(x)} \int_{\beta\left(y_{0}\right)}^{\beta(y)} h_{1}\left(s_{1}, t_{1}\right)\left[f_{1}\left(s_{1}, t_{1}\right)+\int_{\alpha\left(x_{0}\right)}^{s_{1}} \int_{\beta\left(y_{0}\right)}^{t_{1}} h_{2}\left(s_{2}, t_{2}\right) d t_{2} d s_{2}\right] d t_{1} d s_{1}\right\}, \\
& \forall(x, y) \in \Delta .
\end{aligned}
$$

Corollary 2 reduces to Theorem $3.1^{\prime}$ in [13].

\section{Application}

In this section, we apply our result in Theorem 1 to study the retarded Volterra-Fredholm integral equations with two variables, which demonstrates that our result is useful to investigate the qualitative properties of solutions of some retarded Volterra-Fredholm integral equations with two variables.

We consider the retarded Volterra-Fredholm integral equation of the form

$$
\begin{aligned}
v(x, y)= & g(x, y)+\int_{x_{0}}^{x} \int_{y_{0}}^{y} F_{1}\left\{s_{1}, t_{1}, v\left(s_{1}-d\left(s_{1}\right), t_{1}-e\left(t_{1}\right)\right),\right. \\
& \left.\times \int_{x_{0}}^{s_{1}} \int_{y_{0}}^{t_{1}} F_{2}\left[s_{2}, t_{2}, v\left(s_{2}-d\left(s_{2}\right), t_{2}-e\left(t_{2}\right)\right)\right] d t_{2} d s_{2}\right\} d t_{1} d s_{1} \\
& +\int_{x_{0}}^{M} \int_{y_{0}}^{N} F_{1}\left\{s_{1}, t_{1}, v\left(s_{1}-d\left(s_{1}\right), t_{1}-e\left(t_{1}\right)\right), \int_{x_{0}}^{s_{1}} \int_{y_{0}}^{t_{1}} F_{2}\left[s_{2}, t_{2}, v\left(s_{2}-d\left(s_{2}\right), t_{2}-e\left(t_{2}\right)\right)\right] d t_{2} d s_{2}\right\} d t_{1} d s_{1}
\end{aligned}
$$


for all $(x, y) \in\left[x_{0}, M\right] \times\left[y_{0}, N\right]$, where $g, v \in C(\Delta, \mathbf{R})$, $x-d(x) \in C^{1}\left(I_{1}, I_{1}\right)$, and $y-e(y) \in C^{1}\left(I_{2}, I_{2}\right)$ are strictly increasing with $d\left(x_{0}\right)=0, e\left(y_{0}\right)=0, d(x) \geq 0, e(y) \geq 0$, $d^{\prime}(x)<1, e^{\prime}(y)<1$, and $F_{1}, F_{2} \in C(\Delta \times \mathbf{R}, \mathbf{R})$. Let $\alpha(x)=$ $x-d(x), \beta(y)=y-e(y)$; then $\alpha(x), \beta(y)$ satisfy the condition in Theorem 1 and are invertible functions.

The following corollary gives the bound on the solution of (40).

$$
\begin{aligned}
H^{*}(u)= & W_{2}\left\{W_{1}(2 u-k)\right\} \\
& -W_{2}\left\{W_{1}(u)+\int_{\alpha\left(x_{0}\right)}^{\alpha(M)} \int_{\beta\left(y_{0}\right)}^{\beta(N)} E h_{1}\left(\alpha^{-1}\left(s_{1}\right), \beta^{-1}\left(t_{1}\right)\right) f_{1}\left(\alpha^{-1}\left(s_{1}\right), \beta^{-1}\left(t_{1}\right)\right) d t_{1} d s_{1}\right\} \\
& -\int_{\alpha\left(x_{0}\right)}^{\alpha(M)} \int_{\beta\left(y_{0}\right)}^{\beta(N)} E h_{1}\left(\alpha^{-1}\left(s_{1}\right), \beta^{-1}\left(t_{1}\right)\right)\left[\int_{\alpha\left(x_{0}\right)}^{s_{1}} \int_{\beta\left(y_{0}\right)}^{t_{1}} E h_{2}\left(\alpha^{-1}\left(s_{2}\right), \beta^{-1}\left(t_{2}\right)\right) d t_{2} d s_{2}\right] d t_{1} d s_{1}
\end{aligned}
$$

is increasing and $H^{*}(u)=0$ has a solution $c$ for $u>k$. If $v(x, y)$ is a solution of (40) on $\Delta$, then

$$
\begin{aligned}
|v(x, y)| \leq & W_{1}^{-1} \\
& \times\left\{W _ { 2 } ^ { - 1 } \left\{W_{2}\left\{W_{1}(c)+\int_{\alpha\left(x_{0}\right)}^{\alpha(x)} \int_{\beta\left(y_{0}\right)}^{\beta(y)} E h_{1}\left(\alpha^{-1}\left(s_{1}\right), \beta^{-1}\left(t_{1}\right)\right) f_{1}\left(\alpha^{-1}\left(s_{1}\right), \beta^{-1}\left(t_{1}\right)\right) d t_{1} d s_{1}\right\}\right.\right. \\
& \left.\left.\quad+\int_{\alpha\left(x_{0}\right)}^{\alpha(x)} \int_{\beta\left(y_{0}\right)}^{\beta(y)} E h_{1}\left(\alpha^{-1}\left(s_{1}\right), \beta^{-1}\left(t_{1}\right)\right)\left[\int_{\alpha\left(x_{0}\right)}^{s_{1}} \int_{\beta\left(y_{0}\right)}^{t_{1}} E h_{2}\left(\alpha^{-1}\left(s_{2}\right), \beta^{-1}\left(t_{2}\right)\right) d t_{2} d s_{2}\right] d t_{1} d s_{1}\right\}\right\}
\end{aligned}
$$

where $f_{1}(s, t), h_{1}(s, t), h_{2}(s, t), w(s), w_{1}(s)$, and $w_{2}(s)$ are as in Theorem 1. Assume that the function

Corollary 4. Let $k=\max _{(x, y) \in \Delta}\{|g(x, y)|\}$. Suppose that in (40) satisfy the conditions

$$
\begin{gathered}
\left|F_{1}(s, t, x, y)\right| \leq h_{1}(s, t) w(|x|)\left[f_{1}(s, t) w_{1}(|x|)+|y|\right], \\
\left|F_{2}(s, t, x)\right| \leq h_{2}(s, t) w_{2}(|x|),
\end{gathered}
$$
n

where $E=\max _{x \in I_{1}}\left(1 / \alpha^{\prime}\left(\alpha^{-1}(x)\right)\right) \max _{y \in I_{2}}\left(1 / \beta^{\prime}\left(\beta^{-1}(y)\right)\right)<\quad$ Proof. Using the conditions (41), we have $\infty)<\infty$ and $W_{1}, W_{2}, W_{1}^{-1}$, and $W_{2}^{-1}$ are as in Theorem 1 .

$$
\begin{aligned}
& |v(x, y)| \leq|g(x, y)| \\
& +\int_{x_{0}}^{x} \int_{y_{0}}^{y} h_{1}\left(s_{1}, t_{1}\right) w\left(\left|v\left(s_{1}-d\left(s_{1}\right), t_{1}-e\left(t_{1}\right)\right)\right|\right) \\
& \times\left[f_{1}\left(s_{1}, t_{1}\right) w_{1}\left(\left|v\left(s_{1}-d\left(s_{1}\right), t_{1}-e\left(t_{1}\right)\right)\right|\right)\right. \\
& \left.\quad+\int_{x_{0}}^{s_{1}} \int_{y_{0}}^{t_{1}} h_{2}\left(s_{2}, t_{2}\right) w_{2}\left(\left|v\left(s_{2}-d\left(s_{2}\right), t_{2}-e\left(t_{2}\right)\right)\right|\right) d t_{2} d s_{2}\right] d t_{1} d s_{1}
\end{aligned}
$$




$$
\begin{aligned}
& +\int_{x_{0}}^{M} \int_{y_{0}}^{N} h_{1}\left(s_{1}, t_{1}\right) w\left(\left|v\left(s_{1}-d\left(s_{1}\right), t_{1}-e\left(t_{1}\right)\right)\right|\right) \\
& \times\left[f_{1}\left(s_{1}, t_{1}\right) w_{1}\left(\left|v\left(s_{1}-d\left(s_{1}\right), t_{1}-e\left(t_{1}\right)\right)\right|\right)\right. \\
& \left.\quad+\int_{x_{0}}^{s_{1}} \int_{y_{0}}^{t_{1}} h_{2}\left(s_{2}, t_{2}\right) w_{2}\left(\left|v\left(s_{2}-d\left(s_{2}\right), t_{2}-e\left(t_{2}\right)\right)\right|\right) d t_{2} d s_{2}\right] d t_{1} d s_{1} \\
& \leq k+\int_{x_{0}}^{x} \int_{y_{0}}^{y} h_{1}\left(s_{1}, t_{1}\right) w\left(\left|v\left(\alpha\left(s_{1}\right), \beta\left(t_{1}\right)\right)\right|\right) \\
& \times\left[f_{1}\left(s_{1}, t_{1}\right) w_{1}\left(\left|v\left(\alpha\left(s_{1}\right), \beta\left(t_{1}\right)\right)\right|\right)\right. \\
& \left.\quad+\int_{x_{0}}^{s_{1}} \int_{y_{0}}^{t_{1}} h_{2}\left(s_{2}, t_{2}\right) w_{2}\left(\left|v\left(\alpha\left(s_{2}\right), \beta\left(t_{2}\right)\right)\right|\right) d t_{2} d s_{2}\right] d t_{1} d s_{1} \\
& +\int_{x_{0}}^{M} \int_{y_{0}}^{N} h_{1}\left(s_{1}, t_{1}\right) w\left(\left|v\left(\alpha\left(s_{1}\right), \beta\left(t_{1}\right)\right)\right|\right) \\
& \times \\
& \quad\left[f_{1}\left(s_{1}, t_{1}\right) w_{1}\left(\left|v\left(\alpha\left(s_{1}\right), \beta\left(t_{1}\right)\right)\right|\right)\right. \\
& \left.\quad+\int_{x_{0}}^{s_{1}} \int_{y_{0}}^{t_{1}} h_{2}\left(s_{2}, t_{2}\right) w_{2}\left(\left|v\left(\alpha\left(s_{2}\right), \beta\left(t_{2}\right)\right)\right|\right) d t_{2} d s_{2}\right] d t_{1} d s_{1}
\end{aligned}
$$

for all $(x, y) \in \Delta$. From (44), using change of variables, we obtain

$$
\begin{aligned}
& \leq k+\int_{x_{0}}^{x} \int_{y_{0}}^{y} h_{1}\left(s_{1}, t_{1}\right) w\left(\left|v\left(\alpha\left(s_{1}\right), \beta\left(t_{1}\right)\right)\right|\right) \\
& \times\left[f_{1}\left(s_{1}, t_{1}\right) w_{1}\left(\left|v\left(\alpha\left(s_{1}\right), \beta\left(t_{1}\right)\right)\right|\right)\right. \\
&+\int_{\alpha\left(x_{0}\right)}^{\alpha\left(s_{1}\right)} \int_{\beta\left(y_{0}\right)}^{\beta\left(t_{1}\right)} \frac{1}{\alpha^{\prime}\left(\alpha^{-1}\left(s_{2}\right)\right)} \\
& \times \frac{1}{\beta^{\prime}\left(\beta^{-1}\left(t_{2}\right)\right)} h_{2} \\
& \times\left(\alpha^{-1}\left(s_{2}\right), \beta^{-1}\left(t_{2}\right)\right) w_{2} \\
&\left.\times\left(\left|v\left(s_{2}, t_{2}\right)\right|\right) d t_{2} d s_{2}\right] d t_{1} d s_{1} \\
&+\int_{x_{0}}^{M} \int_{y_{0}}^{N} h_{1}\left(s_{1}, t_{1}\right) w\left(\left|v\left(\alpha\left(s_{1}\right), \beta\left(t_{1}\right)\right)\right|\right) \\
& \times\left[f_{1}\left(s_{1}, t_{1}\right)\right. \\
&
\end{aligned}
$$

$$
\begin{gathered}
+\int_{\alpha\left(x_{0}\right)}^{\alpha\left(s_{1}\right)} \int_{\beta\left(y_{0}\right)}^{\beta\left(t_{1}\right)} \frac{1}{\alpha^{\prime}\left(\alpha^{-1}\left(s_{2}\right)\right)} \frac{1}{\beta^{\prime}\left(\beta^{-1}\left(t_{2}\right)\right)} h_{2} \\
\times\left(\alpha^{-1}\left(s_{2}\right), \beta^{-1}\left(t_{2}\right)\right) w_{2}
\end{gathered}
$$$$
\left.\times\left(\left|v\left(s_{2}, t_{2}\right)\right|\right) d t_{2} d s_{2}\right] d t_{1} d s_{1}
$$$$
\leq k+\int_{\alpha\left(x_{0}\right)}^{\alpha(x)} \int_{\beta\left(y_{0}\right)}^{\beta(y)} E h_{1}\left(\alpha^{-1}\left(s_{1}\right), \beta^{-1}\left(t_{1}\right)\right) w\left(\left|v\left(s_{1}, t_{1}\right)\right|\right)
$$$$
\times\left[f_{1}\left(\alpha^{-1}\left(s_{1}\right), \beta^{-1}\left(t_{1}\right)\right) w_{1}\left(\left|v\left(s_{1}, t_{1}\right)\right|\right)\right.
$$$$
+\int_{\alpha\left(x_{0}\right)}^{s_{1}} \int_{\beta\left(y_{0}\right)}^{t_{1}} E h_{2}\left(\alpha^{-1}\left(s_{2}\right), \beta^{-1}\left(t_{2}\right)\right) w_{2}
$$$$
\left.\times\left(\left|v\left(s_{2}, t_{2}\right)\right|\right) d t_{2} d s_{2}\right] d t_{1} d s_{1}
$$$$
+\int_{\alpha\left(x_{0}\right)}^{\alpha(M)} \int_{\beta\left(y_{0}\right)}^{\beta(N)} E h_{1}\left(\alpha^{-1}\left(s_{1}\right), \beta^{-1}\left(t_{1}\right)\right) w\left(\left|v\left(s_{1}, t_{1}\right)\right|\right)
$$$$
\times\left[f_{1}\left(\alpha^{-1}\left(s_{1}\right), \beta^{-1}\left(t_{1}\right)\right) w_{1}\left(\left|v\left(s_{1}, t_{1}\right)\right|\right)\right.
$$$$
+\int_{\alpha\left(x_{0}\right)}^{s_{1}} \int_{\beta\left(y_{0}\right)}^{t_{1}} E h_{2}\left(\alpha^{-1}\left(s_{2}\right), \beta^{-1}\left(t_{2}\right)\right) w_{2}
$$ 


$$
\begin{aligned}
& \times\left(\left|v\left(s_{2}, t_{2}\right)\right|\right) d t_{2} d s_{2} \\
& \left.\times \frac{1}{\alpha^{\prime}\left(\alpha^{-1}\left(s_{2}\right)\right)}\right] d t_{1} d s_{1},
\end{aligned}
$$

for all $(x, y) \in \Delta$. Applying the result of Theorem 1 to the inequality (45), we obtain the desired estimation (39).

\section{Conflict of Interests}

The authors declare that there is no conflict of interests regarding the publication of this paper.

\section{Acknowledgments}

This research was supported by National Natural Science Foundation of China (Project no. 11161018), the NSF of Guangxi Zhuang Autonomous Region (nos. 2012GXNSFAA053009, 2013GXNSFAA019022), the SRF of the Education Department of Guangxi Zhuang Autonomous Region (nos. 201106LX599, 2013YB243), the NSF of Guangdong Province (no. s2013010013385), the Science Innovation Project of Guangdong Province (2013KJCX0125), and the Key Discipline of Statistics of Hechi University of China (no. 20133). The authors are very grateful to the editor and the referees for their helpful comments and valuable suggestions.

\section{References}

[1] T. H. Gronwall, "Note on the derivatives with respect to a parameter of the solutions of a system of differential equations," Annals of Mathematics, vol. 20, no. 4, pp. 292-296, 1919.

[2] R. Bellman, "The stability of solutions of linear differential equations," Duke Mathematical Journal, vol. 10, pp. 643-647, 1943.

[3] I. Bihari, "A generalization of a lemma of Bellman and its application to uniqueness problems of differential equations," Acta Mathematica Academiae Scientiarum Hungaricae, vol. 7, pp. 81-94, 1956.

[4] D. S. Mitrinović, J. E. Pečarić, and A. M. Fink, Inequalities Involving Functions and Their Integrals and Derivatives, Kluwer Academic Publishers, Dordrecht, The Netherlands, 1991.

[5] D. Baĭnov and P. Simeonov, Integral Inequalities and Applications, Kluwer Academic Publishers, Dordrecht, The Netherlands, 1992.

[6] B. G. Pachpatte, Inequalities for Differential and Integral Equations, Academic Press, London, UK, 1998.

[7] O. Lipovan, "A retarded Gronwall-like inequality and its applications," Journal of Mathematical Analysis and Applications, vol. 252, no. 1, pp. 389-401, 2000.

[8] B. G. Pachpatte, "Explicit bound on a retarded integral inequality," Mathematical Inequalities \& Applications, vol. 7, no. 1, pp. 7-11, 2004.

[9] R. P. Agarwal, S. Deng, and W. Zhang, "Generalization of a retarded Gronwall-like inequality and its applications," Applied Mathematics and Computation, vol. 165, no. 3, pp. 599-612, 2005.
[10] W.-S. Cheung, "Some new nonlinear inequalities and applications to boundary value problems," Nonlinear Analysis. Theory, Methods \& Applications A, vol. 64, no. 9, pp. 2112-2128, 2006.

[11] W.-S. Wang, "A generalized retarded Gronwall-like inequality in two variables and applications to BVP," Applied Mathematics and Computation, vol. 191, no. 1, pp. 144-154, 2007.

[12] R. P. Agarwal, C. S. Ryoo, and Y.-H. Kim, "New integral inequalities for iterated integrals with applications," Journal of Inequalities and Applications, vol. 2007, Article ID 24385, 18 pages, 2007.

[13] Q.-H. Ma and J. Pečarić, "Estimates on solutions of some new nonlinear retarded Volterra-Fredholm type integral inequalities," Nonlinear Analysis. Theory, Methods \& Applications A, vol. 69, no. 2, pp. 393-407, 2008.

[14] W.-S. Wang and C.-X. Shen, "On a generalized retarded integral inequality with two variables," Journal of Inequalities and Applications, vol. 2008, Article ID 518646, 9 pages, 2008.

[15] Y.-H. Kim, "Gronwall, Bellman and Pachpatte type integral inequalities with applications," Nonlinear Analysis. Theory, Methods \& Applications A, vol. 71, no. 12, pp. e2641-e2656, 2009.

[16] R. A. C. Ferreira and D. F. M. Torres, "Generalized retarded integral inequalities," Applied Mathematics Letters, vol. 22, no. 6, pp. 876-881, 2009.

[17] W.-S. Wang, Z. Li, Y. Li, and Y. Huang, "Nonlinear retarded integral inequalities with two variables and applications," Journal of Inequalities and Applications, vol. 2010, Article ID 240790, 21 pages, 2010.

[18] W.-S. Wang, R.-C. Luo, and Z. Li, "A new nonlinear retarded integral inequality and its application," Journal of Inequalities and Applications, vol. 2010, Article ID 462163, 9 pages, 2010.

[19] L. Li, F. Meng, and L. He, "Some generalized integral inequalities and their applications," Journal of Mathematical Analysis and Applications, vol. 372, no. 1, pp. 339-349, 2010.

[20] A. Abdeldaim and M. Yakout, "On some new integral inequalities of Gronwall-Bellman-Pachpatte type," Applied Mathematics and Computation, vol. 217, no. 20, pp. 7887-7899, 2011.

[21] W.-S. Wang, "Some generalized nonlinear retarded integral inequalities with applications," Journal of Inequalities and Applications, vol. 2013, article 31, 14 pages, 2012.

[22] H. Zhou, D. Huang, W.-S. Wang, and J.-X. Xu, "Some new difference inequalities and an application to discrete-time control systems," Journal of Applied Mathematics, vol. 2012, Article ID 214609, 14 pages, 2012.

[23] Sh. S. Behzadi, S. Abbasbandy, T. Allahviranloo, and A. Yildirim, "Application of homotopy analysis method for solving a class of nonlinear Volterra-Fredholm integro-differential equations," The Journal of Applied Analysis and Computation, vol. 2, no. 2, pp. 127-136, 2012.

[24] M. Zarebnia, "A numerical solution of nonlinear VolterraFredholm integral equations," The Journal of Applied Analysis and Computation, vol. 3, no. 1, pp. 95-104, 2013.

[25] W.-S. Wang, D. Huang, and X. Li, "Generalized retarded nonlinear integral inequalities involving iterated integrals and an application," Journal of Inequalities and Applications, vol. 2013, article 376, 17 pages, 2013. 


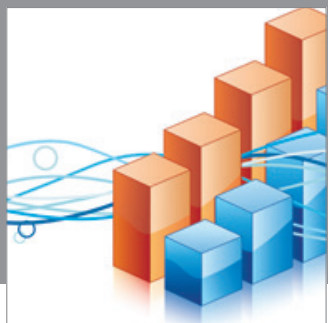

Advances in

Operations Research

mansans

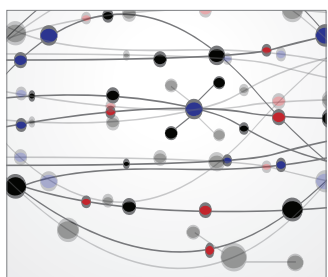

The Scientific World Journal
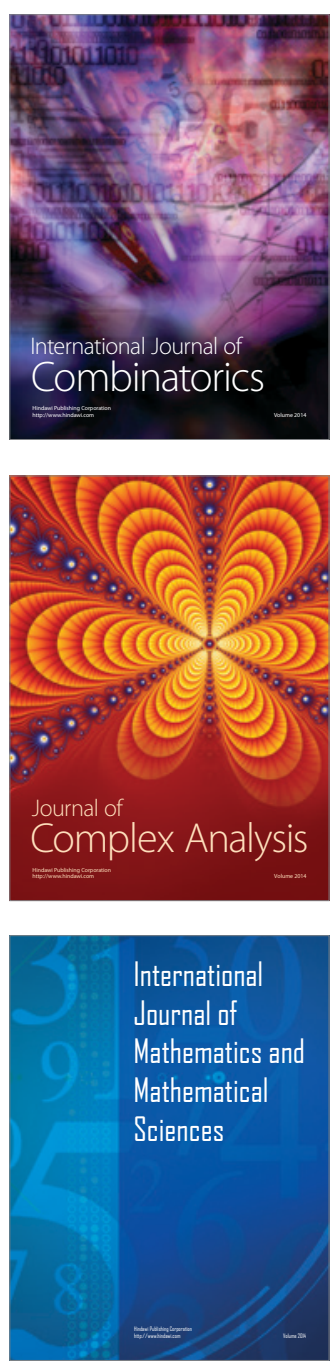
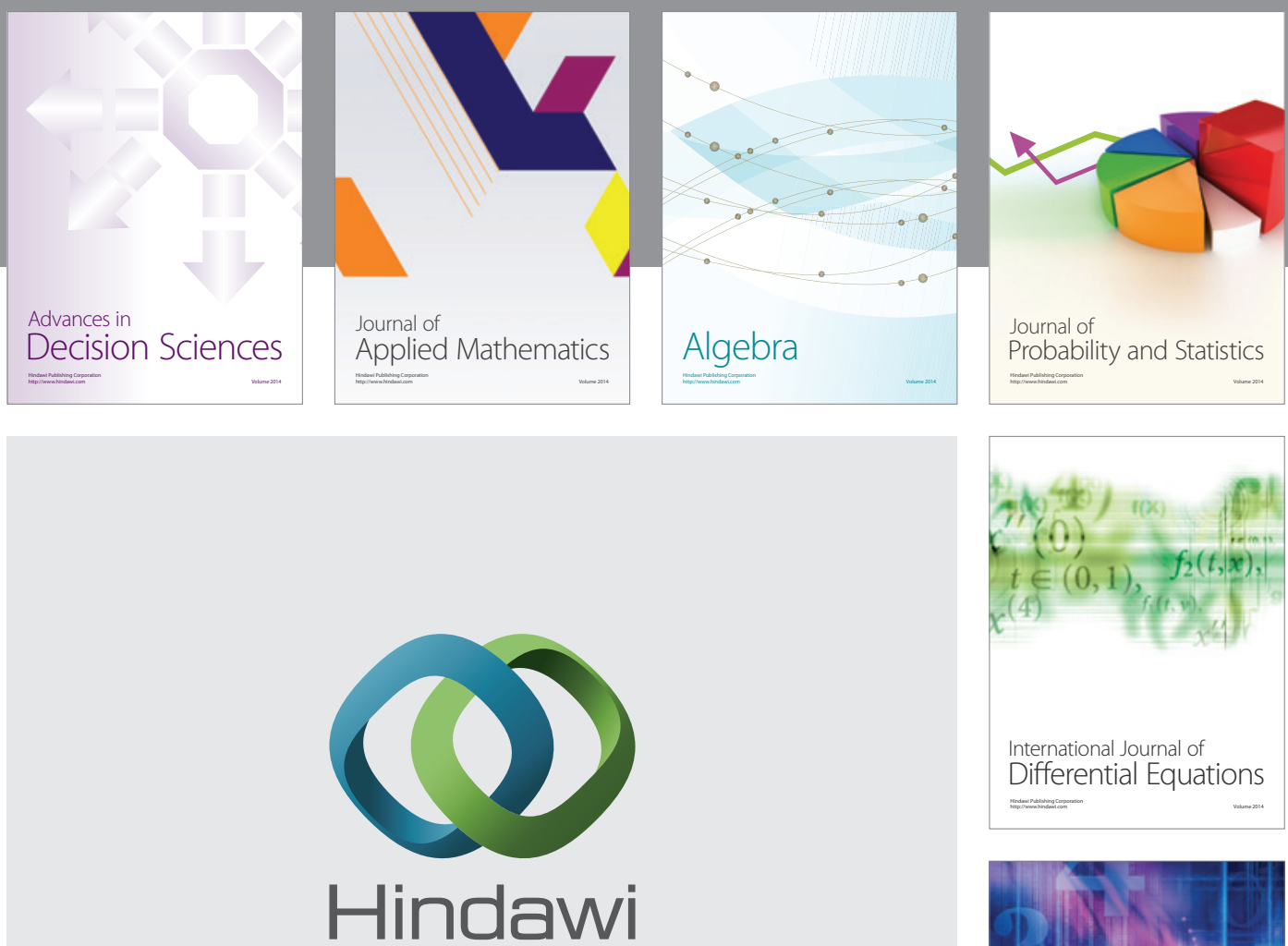

Submit your manuscripts at http://www.hindawi.com


Journal of

Function Spaces

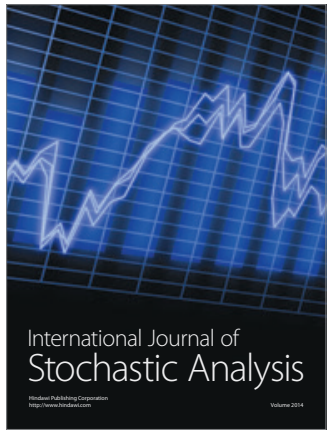

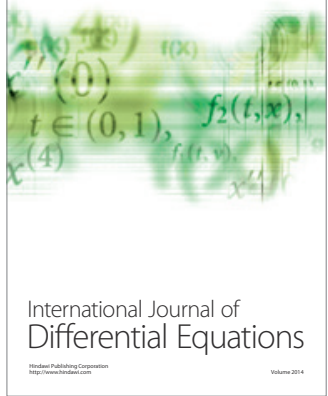
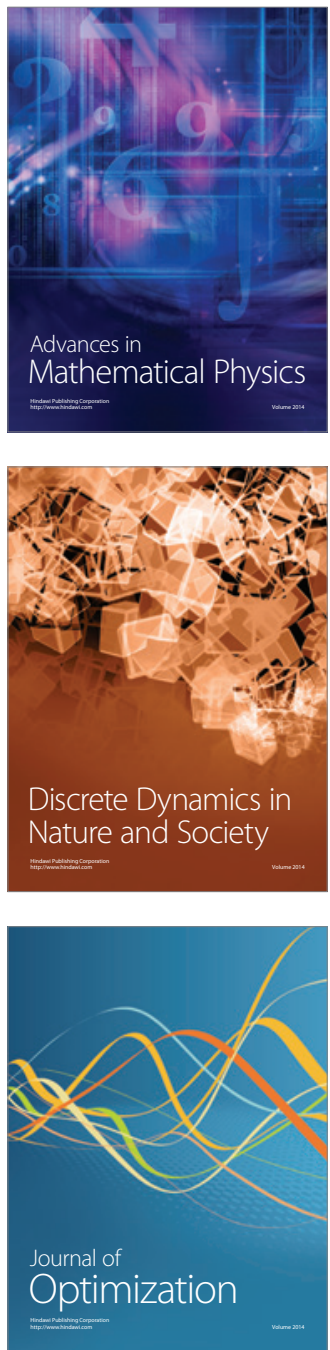